\title{
Comparison between voice quality alteration and dysphagia severity in stroke patients
}

\section{Introduction}

Dysphagia is often found in stroke patients and is associated with clinical complications such as pneumonia, dehydration, and malnutrition, ${ }^{1}$ therefore, besides functional aspects, dysphagia may impact quality of life with emotional, physical, and social implications. $^{2}$

Early detection of dysphagia reduces mortality and admission time, consequently lowering costs with hospitalization, ${ }^{2,3}$ in this perspective, bedside clinical evaluations help decision-making as they are more economic, allow for immediate treatment initiation, and are non-invasive procedures ${ }^{4}$. However, despite commonly employed, the literature lacks consensus concerning the parameters that should be part of bedside clinical evaluations. ${ }^{3,4}$

Voice is produced in the vocal tract and is the result of the interaction between vocal fold vibration and the resonance system, among other roles, the larynx works in phonation and protection of the airways; thus, when the protection mechanism is compromised, voice production may also be impacted..$^{5}$

The presence of dysphonia in stroke patients is common ${ }^{6}$ and figures among the parameters investigated in cases of suspected dysphagia ${ }^{4}$ since the reduction in laryngeal elevation, inefficient glottal closure, and weaker protection of the upper airways are some of the predictive factors for aspiration risk. ${ }^{7}$ Both voice production and deglutition are coordinated systems that require precise interaction among oral, pharyngeal, laryngeal, and respiratory musculatures. ${ }^{8}$

Although neurogenic dysphagia is often associated with "wet voice," laryngeal disorders may also result in voice alterations such as hoarseness, strain, breathiness, roughness, and instability. ${ }^{4,9}$ Clinically, voice quality may provide evidence of the integrity of laryngeal structures and, more specifically, post-deglutition changes in voice quality may indicate the presence of secretion or food in the laryngeal area.Therefore, such changes serve as a sign of possible penetration or aspiration. ${ }^{10}$

In this context, bedside voice quality evaluation as a routine procedure may enhance the investigation of compromised glottis and its repercussions in deglutition of stroke patients.In view of that, this study aimed to investigate the correlation between alterations in voice quality and dysphagia severity in stroke patients.

\section{Methods}

This cross-sectional study was approved by the Research Ethics Committee of the hospital where the research was conducted under protocol 723,678/14. All subjects were duly informed about the research and signed a term of free and informed consent. The inclusion criteria were being 18 years old or more, having a medical diagnostic of ischemic stroke, and having been admitted and diagnosed less than $24 \mathrm{~h}$ earlier. The subjects were excluded if they had other associated neurologic pathologies such as dementia or neurodegenerative diseases, prior speech therapy rehabilitation intervention, or provided incomplete identification data.

\author{
Volume 6 Issue | - 202 |
}

\begin{abstract}
Marcelo Henrique Ferreira,' Jade Zaccarias Bello, ${ }^{2}$ Bruno Francisco de Fraga, ${ }^{3}$ Mauriceia Cassol, ${ }^{4}$ Sheila Tamanini de Almeida ${ }^{5}$

'Speech Therapy Course of the Federal University of Healthcare Sciences of Porto Alegre - UFCSPA - Porto Alegre (RS), Brazil ${ }^{2}$ Graduate Program in Rehabilitation Sciences (Master's Degree), Federal University of Healthcare Sciences of Porto Alegre UFCSPA - Porto Alegre (RS), Brazil

${ }^{3}$ Graduate Program in Rehabilitation Sciences of the Federal University of Healthcare Sciences of Porto Alegre - UFCSPA Porto Alegre (RS), Brazil

${ }^{4}$ Speech Therapy Department of the Federal University of Healthcare Sciences of Porto Alegre, Graduate Program in Rehabilitation Sciences of the Federal University of Healthcare Sciences of Porto Alegre - UFCSPA - Porto Alegre (RS), Brazil ${ }^{5}$ Speech Therapy Department of the Federal University of Healthcare Sciences of Porto Alegre - UFCSPA - Porto Alegre (RS), Brazil
\end{abstract}

\begin{abstract}
Correspondence: Speech Therapy Department, Federal University of Health Sciences of Porto Alegre - UFCSPA Porto Alegre (RS) Brazil, Email sheilat@ufcspa.edu.br
\end{abstract}

Received: October 08, 2020 | Published: February 10, 202 I

The sample consisted in 65 patients recruited through a convenience sample. Patients seen at the stroke unit of philanthropic hospital in Porto Alegre - Brazil, were invited to take part in the study and all of them were evaluated within $24 \mathrm{~h}$ of admission. Most subjects were male, at 38 patients (58.5\%) compared to 27 (41.5\%) females. The mean age was 63.3 years, ranging from 27 to 84 . The age analysis by sex revealed mean age of 60.5 years for the men and 67.1 years for the women.

The patients' files were used to identify the subjects and analyze the study criteria. Next, a brief bedside questionnaire with three yes/ no questions was applied. The questions inquired about the presence of previous alteration in deglutition, presence of other neurological affections, and prior speech therapy.

After that, the subjects underwent two types of evaluations by the speech therapist in the stroke clinic staff: Voice auditoryperceptual analysis was performed using the GRBASI scale ${ }^{11,12}$ while the deglutition evaluation used the Gugging Swallowing Screen (GUSS). ${ }^{13}$

In addition to being internationally employed and acknowledged, the GRBASI scale is the gold standard in voice evaluation and reigns over other methods. The scale assesses the characteristics of overall grade $(\mathrm{G})$, roughness $(\mathrm{R})$, breathiness $(\mathrm{B})$, asthenia (A), strain (S), and instability (I) with scores between 0 and 3 indicating normal and severe levels, respectively. For this study, the auditory-perceptual evaluation was recorded by the bed of each patient at a moment with no excess noise in the room using a Sony ICD-PX440 digital recorder. While lying in bed, the patients were asked to emit the sustained vowel /a/ and count from 1 to 10 at regular frequency and intensity. 
The voice auditory-perceptual analysis was performed by a judge specialized in voice and blinded to the study who has vast clinical experience and develops researches in the area. The judge carried out a joint analysis of the recordings of sustained vowel emission and connected speed, as recommended by the authors of the GRBASI protocol.

The GUSS is validated and standardized for bedside use on stroke patients. It has two steps: the first, called Preliminary Investigation or Indirect Swallowing Test, verifies vigilance status, coughing or throat clearing, saliva swallowing, and voice quality; the second is split into three sub-steps according to the consistency of the food to be evaluated, i.e., semisolid, liquid, and solid.

The GUSS evaluation steps are sequential so that, in order to move on to the next step, maximum score must be reached in the current one. At the end, the results are classified according to the score as: normal deglutition (20), mild dysphagia with a low risk of aspiration (15 to 19), moderate dysphagia with a risk of aspiration (10 to 14), and severe dysphagia with high risk of aspiration (0 to 9 ).
The quantitative variables were expressed as means and standard deviation and the categorical variables, as absolute and relative frequencies. Mann-Whitney test was applied to compare the parameters in the GRBASI scale and GUSS between the sexes. Shapiro-Wilk normality test and Levene's homoscedasticity test were applied. Spearman's coefficient was employed to assess the correlation between the GRBASI scale, GUSS, and age. The correlation among the variables is classified according to the percentage, namely: 0 to $20 \%$ - negligible correlation, up to $40 \%$ - weak, $40 \%$ to $60 \%$ - moderate, $60 \%$ to $80 \%$ - strong, and above $80 \%$ - very strong correlation. A $5 \%$ $(\mathrm{p} \leq 0.05)$ significance level was adopted and the analyses were carried out in the software SPSS version 21.0.

\section{Results}

The distributions of results of the GRBASI scale and the GUSS are presented in Table 1.

Table I Sample distribution regarding the voice and deglutition evaluation results

\begin{tabular}{|c|c|c|c|c|c|c|}
\hline GRBASI parameter & GUSS & Normal & & Mild dysphagia & Moderate dysphagia & Severe dysphagia \\
\hline \multirow[t]{4}{*}{ G } & Normal & II & & 0 & 0 & 0 \\
\hline & Mild & 21 & & 9 & 3 & I \\
\hline & Moderate & 3 & & 5 & 4 & 4 \\
\hline & Severe & 0 & & $\mathrm{I}$ & 0 & 3 \\
\hline \multirow[t]{4}{*}{$\mathrm{R}$} & Normal & 18 & 5 & 0 & 0 & 23 \\
\hline & Mild & 15 & 8 & 4 & 4 & 31 \\
\hline & Moderate & 2 & 2 & 3 & 4 & II \\
\hline & Severe & 0 & 0 & 0 & 0 & 0 \\
\hline \multirow[t]{4}{*}{ B } & Normal & 30 & 6 & 3 & I & 40 \\
\hline & Mild & 5 & 7 & 3 & 4 & 19 \\
\hline & Moderate & 0 & 2 & 1 & 2 & 5 \\
\hline & Severe & 0 & 0 & 0 & I & I \\
\hline \multirow[t]{4}{*}{$A$} & Normal & 28 & 4 & 2 & 3 & 37 \\
\hline & Mild & 6 & 6 & 2 & I & 15 \\
\hline & Moderate & 1 & 4 & 3 & 2 & 10 \\
\hline & Severe & 0 & I & 0 & 2 & 3 \\
\hline \multirow[t]{4}{*}{ S } & Normal & 26 & 14 & 5 & 6 & 51 \\
\hline & Mild & 9 & I & 2 & 0 & 12 \\
\hline & Moderate & 0 & 0 & 0 & 2 & 2 \\
\hline & Severe & 0 & 0 & 0 & 0 & 0 \\
\hline \multirow[t]{4}{*}{1} & Normal & 18 & 2 & 0 & 0 & 20 \\
\hline & Mild & 16 & 12 & 5 & 5 & 38 \\
\hline & Moderate & I & I & 2 & 2 & 6 \\
\hline & Severe & 0 & 0 & 0 & I & I \\
\hline
\end{tabular}

Legend: GUSS, gugging swallowing screen; G, grade; R, roughness; B, breathiness; A, asthenia; S, strain; I, instability 
Dysphagia at any level was found in $75.38 \%$ of the subjects, while $83.08 \%$ exhibited dysphonia. A strong and positive correlation was found between the overall grade $(\mathrm{G})$ of the GRBASI scale and dysphagia in the GUSS. Parameters roughness (R), breathiness (B), asthenia (A), and instability (I) in the GRBASI scale had moderate positive correlation.

A negligible and negative correlation was as found in the comparative analysis between strain (S) in the GRBASI scale and the GUSS (Table 2).

Table 2 Correlation between parameters of the overall voice evaluation scale and the deglutition evaluation scale

\begin{tabular}{lll}
\hline \multirow{2}{*}{ Parameters } & GUSS & $\mathbf{p}$ \\
\cline { 2 - 3 } & Correlation coefficient & \\
\hline $\mathrm{G}$ & 0.637 (strong) $<0,00 \mathrm{I}^{*}$ \\
$\mathrm{R}$ & 0.494 (moderate) $<0,00 I^{*}$ \\
$\mathrm{~B}$ & 0.580 (moderate) $<0,00 I^{*}$ \\
$\mathrm{~A}$ & 0.518 (moderate) $<0,00 I^{*}$ \\
$\mathrm{~S}$ & -0.028 (negligible) \\
$\mathrm{I}$ & 0.549 (moderate) $<0,00 I^{*}$ & 0,823 \\
\hline
\end{tabular}

Legend: GUSS, gugging swallowing screen; $G$, grade; $R$, roughness; $B$, breathiness; $A$, asthenia; S, strain; I, instability

*Significant values ( $\mathrm{p} \leq 0.05)$ Spearman's correlation coefficient (rs)

A regular correlation was found among parameters $\mathrm{G}, \mathrm{R}, \mathrm{B}, \mathrm{A}$, and I and the GUSS with age (Table 3).

Table 3 Correlation between parameters of the voice evaluation scale and the deglutition evaluation scale with age

\begin{tabular}{|c|c|c|}
\hline \multirow{2}{*}{ Parameters } & \multicolumn{2}{|l|}{ Age } \\
\hline & Correlation coefficient & $\mathbf{p}$ \\
\hline G & 0.380 (weak) & $0,002 *$ \\
\hline $\mathrm{R}$ & 0.307 (weak) & $0,013 *$ \\
\hline B & 0.413 (moderate) & $0,001 *$ \\
\hline$A$ & 0.331 (weak) & $0,007 *$ \\
\hline S & 0.038 (negligible) & 0,765 \\
\hline I & 0.385 (weak) & $0,002 *$ \\
\hline GUSS & 0.393 (weak) & $0,001 *$ \\
\hline
\end{tabular}

Legend: GUSS, gugging swallowing screen; G, grade; $R$, roughness; $B$, breathiness; A, asthenia; S, strain; I, instability

*Significant values $(\mathrm{p} \leq 0.05)$ Spearman's correlation coefficient ( $r s)$

\section{Discussion}

The present study found a sample with mean age of 63.3 years and predominance of males $(58.5 \%)$ in a stroke clinic, which matches another study, which found predominance of elderly patients in a hospital of the unified health system. ${ }^{14}$

The high prevalence of dysphagia among stroke patients is in accordance with the literature, which relate aspiration pneumonia as the most common complication in this population. ${ }^{15,16} \mathrm{~A}$ previous study ${ }^{17}$ stated that the incidence of deglutition disorders in stroke patients ranges from 20 to $90 \%$ of the cases, which shows the importance of early diagnosis to ensure dietary safety and to prevent malnutrition and dehydration. ${ }^{18}$

The findings in this study show a strong correlation between alteration in the G parameter of the GRBASI scale and the presence of alteration in the GUSS in $83.08 \%$ of the stroke patients, corroborating the literature, which concludes that vocal changes are easily perceptible in neurological vocal alterations. ${ }^{19}$ The magnitude of the correlation between parameter G and the GUSS was more significant because the overall grade of alteration is the most relevant and most easily noticed marker when voice alteration is present since it reflects the overall impact of disfonia. ${ }^{20}$

Parameters breathiness and asthenia ${ }^{12,13,20,21}$ also had a moderate positive correlation with the GUSS. Breathiness indicates audible air turbulence and escaping air in the voice, which points to glottal inefficiency. ${ }^{22}$ Parameter asthenia is related to voice weakness, indicating possible food entry into the airways, thus impacting air supply and setting off laryngeal action mechanisms, leading to reduced loudness and asthenic phonation..$^{22,23}$

Parameter roughness of the GRBASI scale had a moderate positive correlation with the GUSS. That is significant because such voice alterations may indicate the presence of food bolus in the laryngeal region, which results in reduced mucosal wave and increased irregular vibration. ${ }^{23}$

The moderate positive correlation between the GUSS and parameter instability indicates compromised respiratory and neural support since it is characterized by the unstable vibration pattern of the vocal folds (VF), which becomes noticeable in sustained voice emission. ${ }^{22}$

Only parameter strain had a negative correlation with the degree of dysphagia since strain, in this case, is a protection factor of airways as it favors laryngeal elevation and its occlusion, bringing the VF closer and helping protect against the ingress of foreign objects. ${ }^{19}$

Few studies use standardized voice evaluation scales to detect voice alterations related to dysphagia, nonetheless, since the terms used in the scales are standardized and well-known, overall voice characterization is believed to be a reliable test along with a deglutition evaluation scale. That shows the importance of evaluating voice when dealing with a stroke setting as this parameter is the most reliable marker to characterize dysphonia. ${ }^{24}$

\section{Conclusion}

This study found a strong correlation between the alteration in the overall grade of dysphonia and the severity of dysphagia among stroke patients. The data suggests that the evaluation of parameters involving overall voice alterations is an important tool to be used in diagnosis during bedside hospital care.

\section{Funding}

None.

\section{Acknowledgments}

None.

\section{Conflicts of interest}

The authors declare no conflicts of interest. 


\section{References}

1. Ludlow CL. Central nervous system control of voice and swallowing. $J$ Clin Neurophysiol. 2015;32:294-303.

2. Gaspar MDRDF, Pinto GDSA, Gomes RHS, et al. Avaliação da qualidade de vida em pacientes com disfagia neurogênica. Rev CEFAC. 2015;17:1939-1945.

3. Somasundaram S, Henke C, Neumann-Haefelin T, et al. Dysphagia risk assessment in acute left-hemispheric middle cerebral artery stroke. Cerebrovasc Dis. 2014;37:217-222.

4. Guillén-Solà A, Marco E, Martínez-Orfila J, et al. Usefulness of the volume-viscosity swallow test for screening dysphagia in subacute stroke patients in rehabilitation income. Neuro Rehabil. 2013;33:631-638.

5. Behlau M. Voz: o livro do especialista. Rio de Janeiro: Revinter; 2001. 576

6. Valim MAB, Jurkiewicz AL, Santos RS, et al. Relação do tempo máximo de fonação em pacientes com disfagia secundária a acidente vascular cerebral. Rev Neurocienc. 2013;21:209-215.

7. Santos RRDD, Cola PC, Jorge AG, et al. Correlation between oral and pharyngeal transit time in stroke. Audiol Commun Res. 2015;20:198-202.

8. Ludlow CL. Central nervous system control of voice and swallowing. $J$ Neurophysiol. 2015;32(4):294-303.

9. Martins SRM, de Oliveira DSF. Correlação do grau da disfagia orofaríngea neurogênica com grau da disfonia em idodos: análises correlatas. Rev Pesq Cuid Fundam. 2014;6:1191-1201.

10. Waito A, Bailey GL, Molfenter SM, et al. Voice-quality abnormalities as a sign of dysphagia: validation against and videofluoroscopic data. Dysphagia. 2011;26:125-134.

11. Hirano M. Clinical examination of voice. Springer Verlag. 1981;17:81-84.

12. Dejonckere PH, Remacle M, Fresnel-Elbaz E, et al. Differentiated perceptual evaluation of pathological voice quality: reliability and correlations with acoustic measurements. Rev Laryngol Otol Rhinol. 1996;117:219-224.

13. Trapl M, Enderle P, Nowotny M, et al. Dysphagia bedside screening for acute-stroke patients. Stroke. 2007;38:2948-2952.
14. Rocha MSG, Almeida AC, Abath NO, et al. Impact of stroke unit in a public hospital on length of hospitalization and rate of early mortality of ischemic stroke patients. Arq Neuropsiquiatr. 2013;71:774-779.

15. Resende PDD, Dobelin JB, Oliveira IBD, et al. Disfagia orofaríngea neurogênica: análise de protocolos de videofluoroscopia brasileiros e norte-americanos. Rev CEFAC. 2015;17:1610-1619.

16. Oliveira ARS, Costa AGS, Morais HCC, et al. Fatores clínicos preditores do risco para aspiração e aspiração respiratória em pacientes com acidente vascular cerebral. Rev Latinoam Enferm. 2015;23:216-224.

17. Nunes MCA, Jurkiewicz AL, Santos RS, et al. Correlation between brain injury and dysphagia in adult patients with stroke. Int Arch Otorhinolaryngol. 2012;16:313-321.

18. Albini RMN, Soares VMN, Wolf AE, et al. Conhecimento da enfermagem sobre cuidados a pacientes disfágicos internados em unidade de terapia intensiva. Rev CEFAC. 2013;41:18-23.

19. Valim MAB, Jurkiewicz, AL, Santos RS, et al. Relação do tempo máximo de fonação em pacientes com disfagia secundária a acidente vascular cerebral. Rev Neurociênc. 2013;21:209-215.

20. Klodsinki D, Fadel CBX, Costa FM, et al. Correlação entre sintomas e avaliação perceptivo-auditiva da voz em indivíduos disfônicos. Audiol Commun Res. 2015;20:84-87.

21. Pontes PAL, Vieira VP, Gonçalves MIR, et al. Características das vozes roucas, ásperas e normais: análise acústica espectrográfica comparativa. Rev Bras Otorrinolaringol. 2002;68:182-188.

22. Andrade LGC, Camargo ZA. Estudo da correlação entre qualidade vocal e disfagia pós acidente vascular cerebral: aspectos acústicos, fisiológicos e perceptivos. [dissertação]. São Paulo: Pontifícia Universidade Católica de São Paulo; 2004

23. Warms T, Richards J. Wet voice as a predictor of penetration and aspiration in oropharyngeal dysphagia. Dysphagia. 2000;15:84-8.

24. Gama ACC, Santos LLM, Sanches NA, et al. Estudo do efeito do apoio visualdo traçado espectrográfico na confiabilidade da análise perceptivoauditiva. Rev CEFAC. 2011;13:314-321. 